\title{
The Development and Innovation of the First Supercomputer YH-1 in China: From the Perspective of Technology History
}

\author{
SI Hongwei 司宏伟, ${ }^{*}$ FENG Lisheng 冯立昇 $2 \dagger$
}

(1. Institute for the History of Natural Sciences, Chinese Academy of Sciences, Beijing 100190, China; 2. Institute for History of Science and Technology \& Ancient Texts, Tsinghua University, Beijing 100084, China)

\begin{abstract}
China's first supercomputer capable of 100 million calculations per second was the YH-1, which was independently developed by the Institute of Computer Science at the National University of Defense Technology (NUDT) between 1978 and 1983. YH-1 played an important role in China's national defense construction and national economic development. It made China one of the few countries in the world to successfully develop a supercomputer. Based on original archive documents, interviews with relevant personnel, and an analysis of the technological parameters of the supercomputers YH-1 in China and Cray-1 in the United States, this paper reviews in detail the historic process of the development of $\mathrm{YH}-1$, analyzing its innovation and summarizing the experience and lessons learned from it. This analysis is significant for current military-civilian integration, and the commercialization of university research findings in China.
\end{abstract}

Keywords: history of technology, supercomputer, YH-1, development and innovation

摘 要: 1978 1983 年由国防科学技术大学计算机研究所独立研制的“银河-I”, 是中 国第一台每秒运算 1 亿次的巨型计算机, 使中国成为当时世界上少数能够研制巨型计算 机的国家之一, 为国防建设和国民经济发展作出了特殊贡献。本文通过发掘研制单位原 始档案资料, 采访相关人员, 对比中美巨型计算机研究, 还原银河-I 的研制历程, 分析 其技术创新, 对当下军民深度融合的科技创新路径和高校自主创新、科技成果转化等具 有一定的现实借鉴意义。

关键词：技术史，巨型计算机，银河-I，研制与创新

Received: March 26, 2021. Revised: July 31, 2021.

This article was copyedited by Elizabeth Hughes.

* Research interest: History of computer. Email: 87208120@qq.com

† Research interest: History of technology. Email: fls@mail.tsinghua.edu.cn 


\section{Introduction}

supercomputer, also known as a gigantic computer or a high-performance
computer, refers to a class of computers with the fastest operating speed, largest storage capacity, and overall best performance in any given era. The evaluation standard of a supercomputer evolves over time. This paper uses the term "supercomputer" as it was defined and regulated in the 1980s when the research object was born: "The digital electronic computer with speed of more than 100 million calculations per second, word length of 64 bits, and main memory capacity of 4-16 MB is considered as a supercomputer" (School History Editorial Board of NUDT 1994, 116-117). ${ }^{1}$

China's first supercomputer was the YH-1, developed by the National University of Defense Technology (NUDT) of the Chinese People's Liberation Army (PLA) (Figure 1). The development of YH-1 began in May 1978, and it passed the national appraisal on December 6, 1983. In 1984, the project won the Special National Defense Science and Technology Achievement Award from the Chinese government.

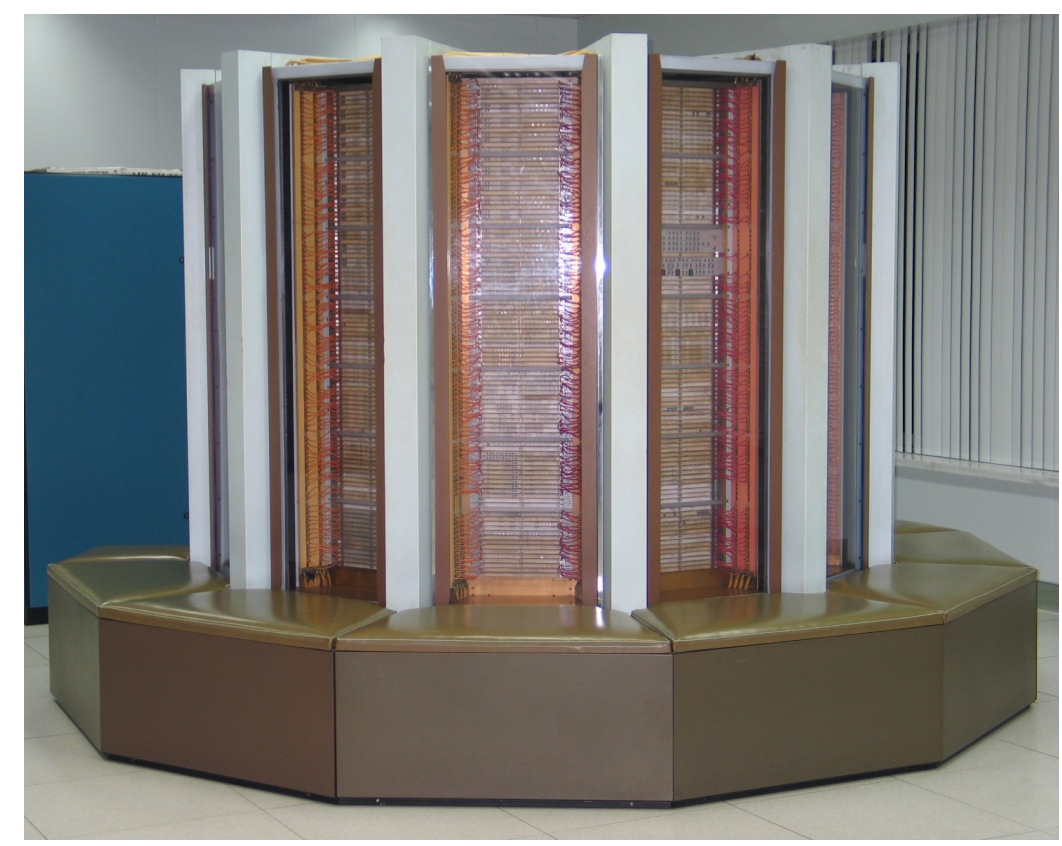

Figure 1: China's first supercomputer YH-1 (courtesy of the Institute of Computer Science, NUDT).

YH-1 was a general-purpose computer that followed the world's advanced

1 “运算速度为每秒 1 亿次以上, 字长达 64 位, 主存容量 $4 \sim 16 \mathrm{MB}$ 的数字式电子计算机才被认为 是巨型计算机。” 
mainstream supercomputer technology. It was based on vector operation, 64-bit word length, and more than 100 million operations per second. Its appearance made China one of the few countries in the world to have successfully developed supercomputers, and it played an important role in the country's national defense and economic development.

At present, the original archives and technical data on the development of YH-1 are closed to the public and have been for a long time. There is a lack of systematic, academic research on the historical background, specific development process, and analysis on the strengths and weaknesses of YH-1. This paper studies the development of China's first supercomputer using existing data, original files, relevant historical documents, and interviews with major researchers.

\section{The background of YH-1 development}

\subsection{The introduction of the development organization}

The Institute of Computer Science at NUDT, which produced YH-1, evolved from the Development Group for Electronic Digital Computers at the Naval Engineering Department of the PLA Military Institute of Engineering in Harbin. In September 1958, this Development Group for Electronic Digital Computers successfully developed China's first tube computer for general military purpose-Model 901 - which could be used on ships, for example (Hu 2016, 385). In July 1964, China's first fully-transistorized general-purpose military computer - Model 441B - was developed at the PLA Military Institute of Engineering in Harbin (Xu 2015, 336). Finally, in December 1976, the institute unveiled China's first large-scale military computer-Model 151 - which could make one million calculations per second (Liu and Li 2006, 114). The achievements in supercomputer development are summarized in Table 1 (Figures 2-4).

Table 1: Achievements in supercomputer development

\begin{tabular}{ccc}
\hline Name of supercomputer & Date & Achievement \\
\hline YH-1 & December 1983 & $\begin{array}{c}\text { China's first supercomputer running 100 million } \\
\text { calculations per second (Si and Feng 2017, 563) }\end{array}$ \\
\hline YH-2 & December 1992 & $\begin{array}{c}\text { China's first supercomputer running one billion } \\
\text { calculations per second (Liu 1996, 23) }\end{array}$ \\
\hline YH-3 & June 1997 & $\begin{array}{c}\text { China's first supercomputer running more than ten billion } \\
\text { calculations per second (Liu 1998, 10) }\end{array}$ \\
\hline YH-X type & June 2000 & Served the national defense and armed forces \\
\hline YH-XX type & December 2006 & Served the national defense and armed forces \\
\hline TH-1 & October 2009 & $\begin{array}{c}\text { China's first quadrillion-times supercomputer (Liu and Si } \\
\text { 2009); ranked the first in the world's TOP500 } \\
\text { supercomputers in November 2010 for the first time }\end{array}$ \\
\hline TH-2 & $\begin{array}{c}\text { China's first supercomputer running more than ten } \\
\text { quadrillion calculations per second (Bai, Wu, and Si 2013); } \\
\text { in the TOP500 list for six consecutive years from 2013 to 2016 }\end{array}$ \\
\hline
\end{tabular}




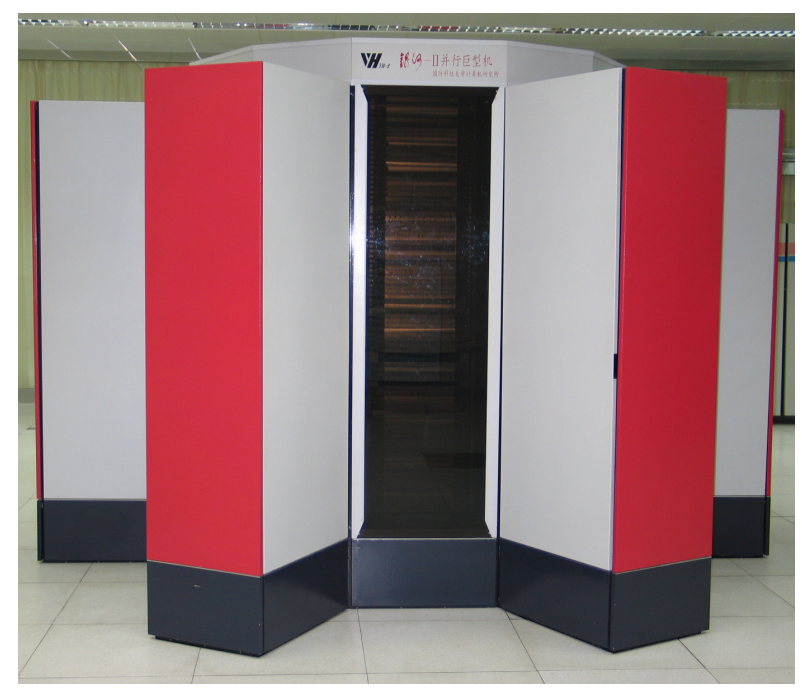

Figure 2: The YH-2 supercomputer (photo by Si Hongwei).

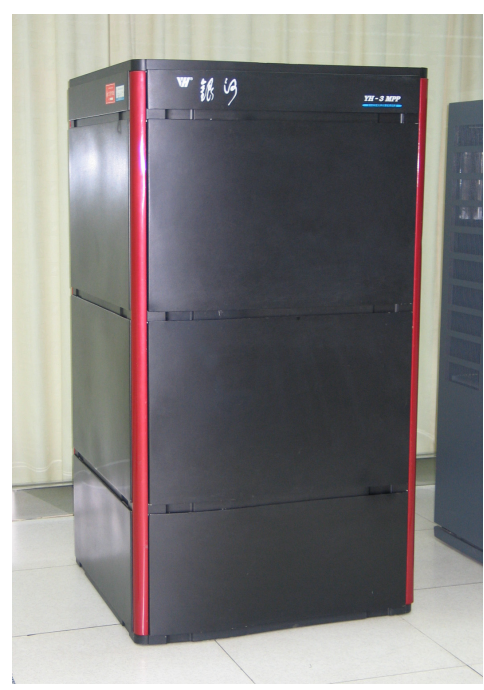

Figure 3: The YH-3 supercomputer (photo by Si Hongwei).

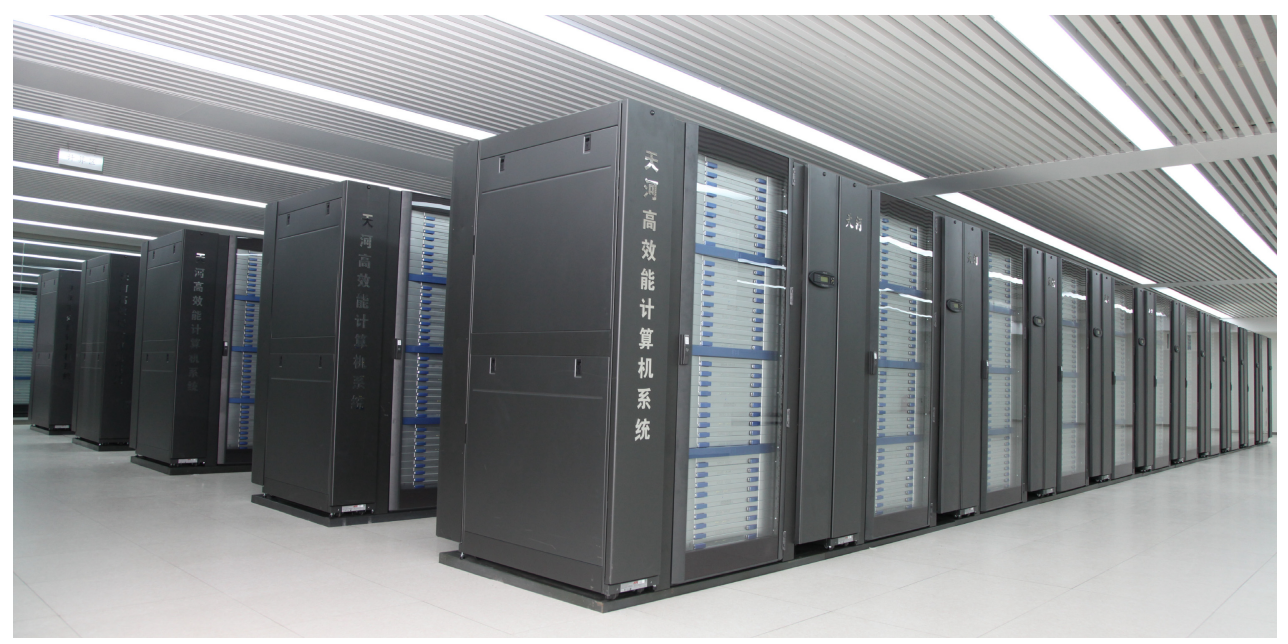

Figure 4: The TH-1 supercomputer (photo by Si Hongwei).

Over the past sixty years, the Institute of Computer Science at NUDT has developed more than forty kinds of super, large, medium, and small computer systems, representing China's ongoing advanced technological contributions. The organization has produced eight academicians of the Chinese Academy of Sciences (CAS) and the Chinese Academy of Engineering, and won a special national defense science and technology achievement award and more than one hundred first prizes at national and ministerial levels. 


\subsection{The reason for the supercomputer development}

Since the founding of the People's Republic of China, the development of the atomic bomb and the hydrogen bomb had become a national development priority, and the computer was critical for the successful completion of the Two Bombs project (两弹工程).

As early as the 1950s, the United States had begun using the computer UNIVAC, which could perform 2000 operations per second, and the IBM-704, with 20,000 operations per second, to design the first thermonuclear weapon. In 1964, it developed a small-scale strategic nuclear warhead using the large computer CDC-6600, which could complete 2 million operations per second. In the late 1960s, the US designed the Spartan Anti-Ballistic Missile with the CD C7600, with 10 million operations per second. After the Cray company successfully launched the Cray-1 supercomputer with 100 million operations per second (Figure 5), the US weapons research department used it to enhance safety mechanisms on nuclear warheads (Murray 1997, 47-50).

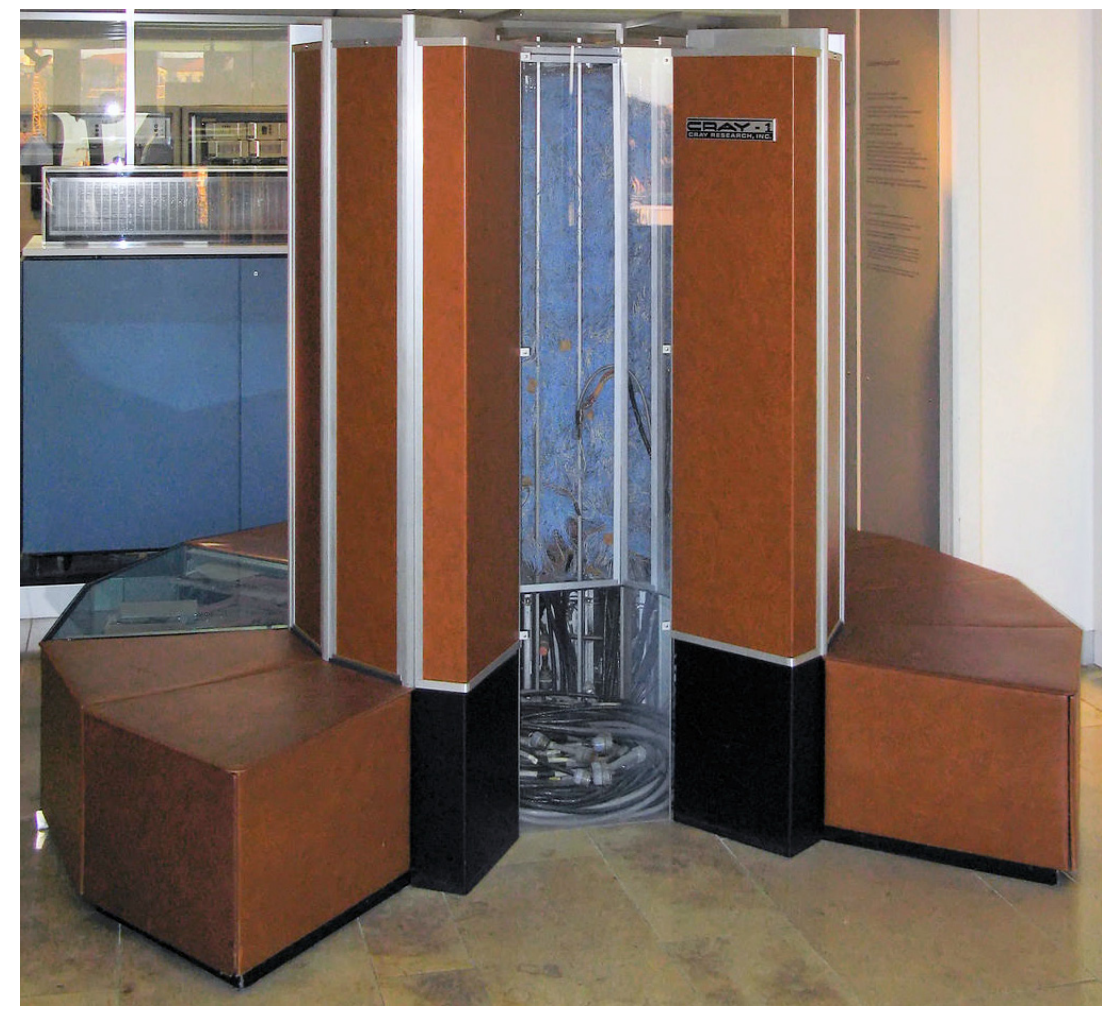

Figure 5: The Cray-1 supercomputer. ${ }^{2}$

2 https://en.wikipedia.org/wiki/File:Cray-1-deutsches-museum.jpg 
During the initial period of China's computer industry, the country received great assistance from the Soviet Union. The USSR sent experts to guide the implementation of China's computer technology plan, establish computer research institutions, and train computer technicians, but they also allowed China to duplicate the M-3 and BESM-II computers. It was a successful technology transfer, which laid a good foundation for China's independent development of computers. By duplicating the M-3 and the BESM-II, Chinese engineers mastered computer design and manufacturing techniques. In 1958 and 1959, they completed two computers - the 103 and 104-the first small electronic tube computer and the first large general-purpose electronic digital computer, respectively, in China. The first 103 and 104 computers were handed over to the military department for use in the development of China's first-generation nuclear weapons (Zhang and Zhang 2007).

The development of China's second-generation strategic nuclear weapons started in the mid-1970s. Commission of Science and Technology for National Defense (COSTND) and the Second Ministry of Machine Building Industry assigned the task of to the Ninth Academy of Engineering, specifically commissioning a more powerful and miniaturized hydrogen bomb, which then became a neutron bomb. The primary boost (especially the gas boost) of a neutron bomb involves a series of complicated physical processes, which are carried out under extremely high temperatures and high pressure. The pressure is as high as millions of atmosphere and the temperature is as high as tens of millions of degrees centigrade. The huge energy of nuclear explosion is released in microseconds. In a nuclear test, it is very difficult to measure the detailed reaction process inside the nuclear weapon. The mathematical model that described the nuclear reaction is a set of unsteady nonlinear partial differential equations. The solution to these equations offers detailed images, quantitative data, and the whole process of nuclear explosion. However, there is no way to find the analytical solution to such equations, so we can only resort to the numerical method to solve it, that is, to carry out large-scale numerical calculations. At the same time, the large-scale scientific calculation is used to simulate the nuclear explosion process and deduce the energy release effect of nuclear devices under different structures and conditions, which can realize the design and improvement of nuclear weapons to a certain extent. The general large-scale computers were incapable of computing at this level, so it was urgent to develop supercomputers with a computing speed of more than 100 million calculations per second.

It can be seen that the development of China's nuclear weapons, especially the second-generation strategic nuclear weapons, is the primary catalyst for the development of supercomputers. Zhang Zhenhuan 张震寰, former deputy director of COSTND, said at China's Supercomputer Research and Development Conference, "Without supercomputers, there would be no second-generation sophisticated 
weapons. If the second-generation sophisticated weapons could not be produced, there would be no way to solve the problem of the neutron bomb, multiple warheads, and miniaturization. If we could not develop a supercomputer with a speed of more than 100 million calculations per second or second-generation weapons, we would delay state affairs of China." 3

\subsection{Accumulation and preparation}

The PLA Military Institute of Engineering in Harbin, established in September 1953, received great help from the advisory group of Soviet experts in the early stages, including assistance in the development of computer technology. With the knowledge gained from the Soviets, the institute successfully developed a series of small, medium, and large computers, such as models 901, 441B, and 151, each model improving on its predecessor's performance capabilities. In 1970, new national defense demands motivated officials to move the institute to Changsha at which point its name was changed to the Changsha Institute of Technology (CIT) (School History Editorial Board of NUDT 1993, 7-169).

In the autumn of 1972, as the need to develop the second-generation strategic nuclear weapons and enhance national defense strength urgently escalated, COSTND ordered the head of the Institute of Computer Science at CIT (the predecessor of the Institute of Computer Science at NUDT), Ci Yungui 慈云桂 (1917-1990), to draft a report to the central special committee, suggesting that the development of a supercomputer be included in China's major engineering project goals.

The senior leaders of the party, government, and army of China recognized the great importance of supercomputers in national defense and military industry. Especially after Deng Xiaoping's 邓小平 comeback in March 1973, supercomputer research was relatively unimpeded by the "Cultural Revolution." At the same time, the successful development of the Model 151 computer with one million instructions per second laid a foundation for the development of the supercomputer. Ci Yungui's proposal was soon approved, and the Institute of Computer Science at CIT began the pre-research and technical accumulation of the supercomputer.

In October 1975, Zhang Aiping 张爱萍, then director of COSTND, ordered the established domestic computer research centers to conduct a national survey. $\mathrm{Ci}$ Yungui was the leader of the survey group and focused his argument supporting development of the supercomputer on high demand, the production situation, and

3 “没有亿次巨型机, 就没有第二代尖端武器, 第二代尖端武器搞不出来, 中子弹、多弹头、小型 化就没法解决, 拿不出亿次机, 拿不出第二代武器, 就会贻误国家大事。”

“Yinhe-I guojia jiandinghui cailiao" 银河-I 国家鉴定会材料 [Materials of YH-1 on National Appraisal Committee]. Archives of College of Computer Science and Technology, NUDT, KTYH1001. 
level of domestic components and external equipment. However, due to interference from the "Gang of Four," Deng Xiaoping was again ousted from office in April 1976, and this work was forced to stop halfway. In September 1977, after the end of the "Cultural Revolution," Zhang again instructed Ci to dispatch core technicians to conduct research on the supercomputer and submitted the resulting report to COSTND. On November 14, 1977, COSTND, in turn, sent a report on the development of the first supercomputer in China to the Central Committee of the Communist Party of China (CCCPC). It was approved on November 26.

\section{The development history of YH-1}

On March 4, 1978, Deng Xiaoping, who had been appointed vice chairman of the Military Commission of the CCCPC and chief of the general staff, presided over the meeting on the deployment of the supercomputer and tasked NUDT with its development. (In that same year, CIT had been incorporated into the PLA and renamed the National University of Defense Technology. ${ }^{4}$ ) In May 1978, COSTND held a conference in Beijing to demonstrate the program and build cooperation in supercomputer research. It was determined that it would take about six years and cost about 200 million yuan to develop a supercomputer with 100 million calculations per second. The task's codename was the "785 project."

\subsection{Technical command system}

The 785 project leading group was established at NUDT, and Ci Yungui was appointed as chief designer and team leader. $\mathrm{Ci}$ was already a renowned computer expert, educator, and member of the Academic Divisions of CAS. He was born in Tongcheng County, Anhui Province (Figure 6), and graduated from the Department of Mechanical and Electrical Engineering of Hunan University in 1943. He pursued his postgraduate studies at the Radio Research Institute of Tsinghua University (THU). After graduation, he worked at the Physics Department of THU as an assistant and lecturer. In 1954, he was transferred to the PLA Military Institute of Engineering in Harbin, and served as director of the radar teaching and research section of the Naval Engineering Department. He was appointed as director of the Electronic Computer Department in 1966. He was then director of the Institute of Computer Science at CIT, later NUDT, beginning in 1970. He had been engaged in the research and development of China's electronic computers for a long time and had overseen the development of China's first

4 “Deng Fuzhuxi zai tingqu jisuanji wenti huibao shi de zhishi” 邓副主席在听取计算机问题汇报 时的指示 [Vice President Deng's Instructions When Listening to the Report on Computer Problems]. Archives of College of Computer Science and Technology, NUDT, ZYJ-1-1-5. 
dedicated digital computer with electronic tubes (Model 901), a fully-transistorized military computer (Model 441B), and a military computer that could complete one million operations per second (Model 151-3/4).

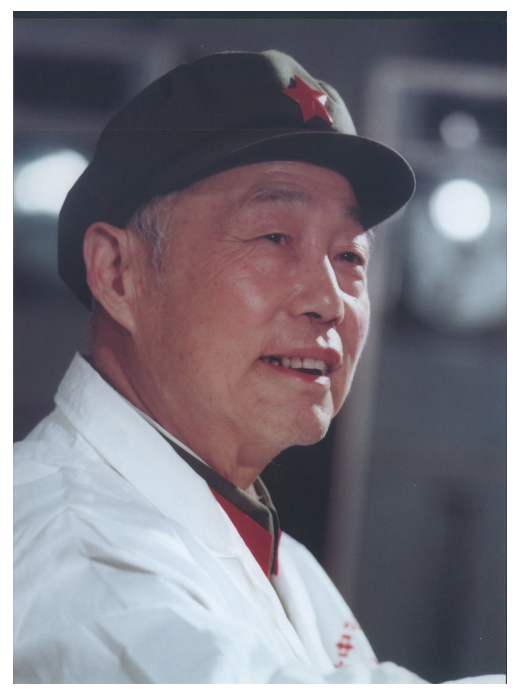

Figure 6: YH-1 chief designer Ci Yungui (courtesy of Ci's family).

The 785 project was very difficult in technology, complex in system, and wide in scope of cooperation. In order to complete the task on time with high quality, the engineering leading group adopted the scientific method of system engineering to establish the technical command system. Ci selected an impeccable team, including Hu Shouren 胡守仁, Wang Zhenqing 王振青, Chen Huowang 陈火旺, Chen Fujie 陈福接, Zhou Xingming 周 兴铭, Zhou Diji 周堤基, Meng Qingyu 孟庆余, and Zhang Defang 张德芳.

Wang Zhenqing, head of the overall hardware team for the supercomputer, and Zhou Xingming, head of the host system and director of the general control office, led the team to develop the hardware system, including the command control office, operation control office, storage control and circuit research offices, automation research office, mechanical structure and technology research office, power supply system research office, and the peripheral system and peripheral equipment research office.

When the overall software team of the supercomputer was established, Ci served as team leader, while Chen Huowang and Meng Qingyu served as deputy group leaders. Later, Chen became the team leader, and Meng was appointed director of the software research office.

\subsection{The development stages}

The research and development of YH-1 between May 1978 and December 1983 can be 
divided into six stages (Table 2).

Table 2: YH-1 development stages

\begin{tabular}{ll}
\hline \multicolumn{1}{c}{ Time } & \multicolumn{1}{c}{ Main work } \\
\hline May-December 1978 & Overall scheme demonstration and project preparation \\
October 1978-March 1980 & Experiment, logic design, plug-in engineering, and model machine development \\
May 1980-July 1981 & Production of the host system \\
March-December 1981 & Debugging stage of supercomputer hardware system \\
August 1979-June 1983 & Development and joint debugging of software system \\
April-December 1983 & National technical examination and appraisal stage \\
\hline
\end{tabular}

\subsubsection{Overall scheme demonstration and project preparation stage}

At the aforementioned COSTND conference in May 1978, several vital decisions were made: a dual processor scheme to be completed in two phases was adopted; it was decided to introduce certain components; and it was resolved that the peripheral machines and external equipment would be developed by domestic cooperation. In July 1978, COSTND held the 785 Engineering Software Cooperation Meeting in Changsha, which implemented the cooperation tasks and suggested instruction-level compatibility between $\mathrm{YH}-1$ and Cray-1. After consulting experts and users and undertaking an in-depth investigation into the development and use of supercomputers at home and abroad, they modified and finalized the overall scheme of YH-1 on the basis of multi-party investigation and demonstration. In view of the difficulties in producing peripheral and external equipment in China, it was ultimately decided to import them from abroad.

\subsubsection{Experiment, logic design, plug-in engineering, and model machine development stage}

In order to keep the design theory in line with the high-speed and high-quality index, the researchers carried out substantial theoretical research and experiments: they put forward the design scheme; determined the logic design principle, implementation technology, and production process; and verified its feasibility and effectiveness through the development of a model machine, which laid a reliable foundation for the entire system.

In the beginning of 1980, the development of the model machine was completed. In March 1980, COSTND held a report meeting on the YH-1 model machine development in Changsha. The success of the model machine development showed that a series of problems, such as theory and technology difficulties and production technology in the hardware development of supercomputer, had been overcome. The production line of 
the supercomputer had been formed in the computer factory of NUDT.

\subsubsection{Production stage of the host system}

The production of the YH-1 host system lasted for fifteen months. Quality control, technology specification, and matching components were the main tasks in this stage. At that time, it was very difficult for the supercomputer to produce a large-area multilayer printed circuit board, because of the high-density packaging, and the applied technology, such as winding and soldering, was extremely difficult. The related domestic factories did not have the capacities for this formidable task. NUDT decided to undertake the project and set up the printed board workshop, welding workshop, and machining workshop, and established the production department, equipment department, and technical department. Over one hundred dedicated workers overcame temperature and humidity extremes in addition to delicate manual labor to successfully complete production.

\subsubsection{Debugging stage of supercomputer hardware system}

In this stage, three problems were solved: first, the plug-in, the bottom plate, the welding point, and the winding point were thoroughly and carefully checked; second, workers analyzed plug-in testing and developed the testing equipment in line with the actual conditions of the supercomputer, which could be remarkably harsh; finally, tools and methods were debugged.

In order to ensure the quality of the supercomputer hardware, the 785 project leading group founded a quality-control group and product-inspection group, established a strict system of post responsibility, and implemented the whole-process quality-tracking control. The researchers also developed a set of computer automatic test systems to complete the $\mathrm{YH}-1$ plug-in board test task, greatly improving the work efficiency and test accuracy. In the past, it took one year or even several years to debug computers with one million operations per second while only sixty-six days were needed to conduct debugging, joint debugging, correctness debugging, and appraisal for YH-1.

\subsubsection{Development and joint debugging stage of software system}

The software development and debugging of $\mathrm{YH}-1$ ran through almost the whole process of the project. In the face of the rapid development of software beyond China and the fact that domestic software standards remained relatively weak, decisionmakers were keenly aware of the importance of research and development of supercomputer software, even if it took great efforts. In this stage, the following tasks

were finished: mobilizing personnel to enrich the software development team; organizing horizontal cooperation in different forms; realizing software engineering 
and standardization; advancing the development of simulators and debugging tools, seeking more time, and shortening the development cycle; ensuring the agreement and coordination of software and hardware, as well as the approval of software design and the inspection and assessment of stage results.

A large software development team of more than one hundred researchers had successfully developed the two-million-line YH-1 system software that was complete in functions, easy to use, and strong in scalability. At the same time, it was compatible with Cray-1.

\subsubsection{National technical examination and appraisal stage}

Supercomputer assessment was composed of user assessment and national technical examination. In order to ensure that the 785 project met the high standards of the design objectives, user technical assessment was organized before national technical examination. In June and September 1982, YH-1 was examined for correctness and reliability of the software and hardware of the whole system, which laid a good foundation for the national examination. In September 1983, Commission of Science, Technology and Industry for National Defense (COSTIND) ${ }^{5}$ established a working committee of the National Technical Appraisal Group of YH-1 in Changsha, improved the technical appraisal outline and implementation rules, formed seven appraisal groups, and completed the final preparation for the National Appraisal Meeting in December 1983.

\subsection{Disclosure of several key historical facts}

\subsubsection{The process of the final overall scheme referring to Cray-1}

At first, the researchers orientated their work on the vector supercomputer TI-ASC developed by Texas Instruments in 1972 and STAR-100 launched by Control Data Corporation in 1973. They made two general plans based on these two examples and almost finalized the plans. At this time, Liu Degui 刘德贵, an alumnus of NUDT working at the Fourth Ministry of Machine Building Industry, procured several advertising materials for the latest supercomputer Cray-1 of the United States in $1976 .{ }^{6}$

The novel and concise design idea of Cray-1 immediately attracted Ci's attention. After analysis and research, he thought that it was of great reference value. Overnight, he made a new concept of the preliminary overall plan for the development of China's supercomputer, which was based on Cray-1 design ideas and suitable for China's

5 In 1982, COSTND and other institutions jointly formed COSTIND.

6 Chen Yuanxing 陈元兴, Zhou Xingming 周兴铭, and Zhang Defang 张德芳. “Kelei-1 juxing jisuanji xitong zongshu" 克雷-1 巨型计算机系统综述 [Overview of the Cray-1 Supercomputer System]. Archives of College of Computer Science and Technology, NUDT, KTYH-I012/6. 
national conditions. In early January 1978, Ci handed over Cray-1 data to the entire team, who were thoroughly impressed by the report. At this time, the overall scheme demonstration meeting was only four months away.

Because of the extreme simplicity of Cray-1 advertising materials, $\mathrm{Ci}$ immediately organized personnel to search for more detailed information, and they found CRAY-1 Hardware Reference Manual (No. 2240004, November 1977) by CRAY Research, Inc. from the reference office of the Institute of Computing Technology at CAS.7 The manual consists of over two hundred pages and comprises six parts: Cray-1's introduction, physical organization, computation section, instructions, memory section, and input/output section. The appendix was also provided with data tables and illustrations. This reference manual was attached to the Cray-1 supercomputer sold by Cray Research. It does not involve the core key technologies of the system and the technical details of how the system is implemented, but is rather the equivalent to an instruction manual open to its users. Ci Yungui and his team members repeatedly analyzed and studied the data and determined Cray-1's design idea and implementation methods to be more advanced and unique than their initial examples. They therefore decided to give up their initial plan, which had been completed after eight months of hard work, and design a new overall scheme based on Cray-1 that conformed to China's national conditions and was compatible with international mainstream supercomputers ( $\mathrm{Ci}$ and $\mathrm{Hu}$ 1980).

Although the new overall scheme referred to the design idea of Cray- 1 of the United States, the key technical details could not be mastered due to the lack of hardware drawings, thus making it difficult to understand its architecture. Furthermore, many technologies, such as the special ultra-high-speed bipolar logic device and the highdensity process assembly technology combined with liquid cooling technology, could not be achieved at that time. The situation was made more complicated by the fact that components and parts of the corresponding equipment could not be purchased. Using the national and institutional resources at hand, researchers carried out a series of bold independent innovations based on the plan, including: a full pipeline supercomputer architecture with bi-vector array, which improved the parallelism and the actual operation speed of the supercomputer; the main memory structure of the prime module conflict-free access that improved the vector reality of the main memory; and selecting high-speed dynamic MOS devices as the main memory, which was a globally leading choice and met user requirements for main memory capacity. Additionally, a wedge-shaped columnar tube guide structure was designed and an equal pressure short air path parallel ventilation was implemented in order to avoid using the precarious liquid cooling technology. Finally, the operating system, compiler system,

7 CRAY-1 Hardware Reference Manual (2240004). Archives of College of Computer Science and Technology, NUDT, KTYH-I012/1. 
application software, and I/O software were independently developed with great difficulty.

\subsubsection{The history of innovative bi-vector array architecture}

After first studying YH-1 supercomputer architecture, researchers had proposed three technical solutions: multi-processor parallel architecture, dual CPU architecture sharing main memory, and single CPU architecture with bi-vector array. After careful deliberation, the multi-processor parallel architecture was considered too advanced and unattainable, which was not in line with China's reality, and it was rejected by Ci.

The other two plans were better suited for the difficult task of making a supercomputer capable of 100 million instructions per second under the limitations of component levels in China. However, with further research, it was found that if the dual CPU architecture were adopted, it was necessary to develop a parallel processing system, a cutting-edge technology. The system was far too complicated to attain by the deadline, especially considering the technical strength required. Thus, the overall team began to consider the single processor scheme.

Traditional computers use scalar computing mode, which can only process one operand or a pair of operands. A vector computer can process the uncorrelated operands at the same time. Compared with a scalar computer, a vector computer is more suitable for large-scale scientific computing. Therefore, Ci proposed to make $\mathrm{YH}$ 1 into a vector supercomputer. The architecture was changed to a single processor system, which required all components, especially the central processing unit, to have stronger performance abilities. Through repeated calculation and given the level of existing components, researchers believed that if the ordinary single vector machine mode was used, it could not reach 100 million calculations per second.

From August to September 1978, Ci organized engineering and technical personnel to study and discuss the advanced technical experience at home and abroad, especially the extremely limited data of Cray-1 supercomputer, and creatively proposed the bivector array architecture. Bi-vector array architecture is a further optimization of the architecture of a common vector machine. A vector operation is carried out in a dual array operation unit, and two sets of data overlap operations are performed simultaneously. Two results can be obtained per beat, and multiple vector operations can be executed in different functional units in parallel. The YH-1 mainly aimed to serve such units as the Ministry of Nuclear Industry. The main task of computing was to solve large-scale computational problems that can only be solved by supercomputers completing more than 100 million operations per second. According to the survey, more than $75 \%$ of these problems were vector operations. The scheme using bi-vector array architecture was suitable for this kind of large-scale scientific computing. At the same time, advanced technologies (such as multi-functional components, full 
pipelining, parallel computing, and distributed structure) also proved to be applicable.

Therefore, $\mathrm{Ci}$ led the team to make major changes to the overall scheme reported at the 785 project meeting. On October 7, 1978, the design of the architecture in the overall demonstration of the supercomputer was completed, and the single processor bi-vector array architecture scheme was selected. In December 1978, COSTND held the 785 project scheme approval meeting in Beijing, and accepted the single processor, bivector array, and full pipeline architecture scheme.

Interestingly, three kinds of supercomputers developed in Japan in the 1980s all adopted bi-vector array architecture, while the new system Cray-MP based on Cray-1 adopted the dual processor system in 1983. "This coincidental fact shows that science is without boundaries and that Chinese people can make a difference" (Zhou and Zhao $2005,44) \cdot{ }^{8}$

\subsubsection{YH-1 was not all localized}

In the initial phases of research into $\mathrm{YH}-1$, researchers imagined that the whole system, including components, raw materials, peripherals, and external equipment, would be made in China. NUDT was responsible for the design and research of the whole system and the development of the main engine. The front-end machine and external equipment were developed by other teams in China according to the design requirements of $\mathrm{YH}-1$. After extensive research throughout the country, which included recording users' opinions and investigating the use of existing supercomputers like Cray-1 abroad, the team realized that the quality of some domestic components was lacking, especially the high-speed circuit and some key raw materials, such as copper-clad laminate for producing multilayer printed board. It was difficult to meet the requirements of engineering quality, and there were also many difficulties in processing peripheral machines and external equipment in China. If the original plan remained unchanged, the task could not be completed on time; even if the machine was developed, it would be difficult for its engineering quality to meet the design requirements.

Ci made a bold decision, challenging his entire staff to emancipate their minds and abandon the one-sided understanding of "localization that even every screw must be made in China." As the country was undergoing the reform and opening-up post"Cultural Revolution," Ci proposed taking advantage of broadening trade agreements; he proposed, " [to] firmly adopt those domestic products that have been produced in China and have passed the quality standard; those absent from China or without quality assurance shall be imported from foreign countries temporarily." 9 Not only did

8 “这种不谋而合的事实说明, 科学是不分国界的, 中国人是能够有所作为的。”

9 “国产化, 就连每一个螺丝钉也得要中国制造的才行。” “采用两条腿走路的方针, 凡国内已经生

产, 而且质量过关的, 坚决采用国内的产品; 凡国内没有或是不能保证质量的, 则暂时从外国引进。” 
this change save time and money for the whole project of $\mathrm{YH}-1$, but it also ensured the high level of the whole system performance. Thus, a scientific research path with Chinese characteristics was found, which was independent, self-reliant, emphasized importation and absorption, and fitted China's national conditions. COSTND set up a special procurement agency to send personnel in multiple dispatches overseas to purchase key components, peripheral machines, and other equipment. In coordination with the General Administration of Customs and Guangzhou Military Region, COSTND managed to purchase a number of integrated circuit chips and highperformance small computers from abroad.

It is worth noting that "considering the cycle and cost of the project, it is a common practice in the field of international computer engineering to purchase non-core components. A large number of parts and even peripheral equipment of Cray-1 supercomputer in the United States are also purchased" (Zhao 2012, 53). ${ }^{10}$

\subsection{The national appraisal results}

In January 1982, the hardware debugging of the 785 project's host system was completed. Zhang Aiping, then vice premier of the State Council, named it the $\mathrm{YH}-1$ supercomputer "Yin He 银河" (the Galaxy). On December 6, 1983, YH-1 successfully passed the national appraisal. The certificate stated,

YH-1 is the first supercomputer system with 100 million calculations per second developed by China. The system is stable and reliable, and the software is relatively complete. Its main technical indexes all meet or exceed the requirements of the appraisal outline. It has reached the domestically advanced level, and some aspects have reached the international level. Its successful development fills in the blank of domestic supercomputer and marks China's entry into the ranks of the world's research and development of supercomputers. ... YH-1 will soon become a powerful computing tool for China's strategic weapons development, aerospace vehicle design, national economic prediction and decision-making, energy development, weather forecast, image processing, information analysis, and various scientific research. ${ }^{11}$

Ci Yungui 慈云桂. “'Qibawu' gongcheng yinhe yiciji yanzhi baogao” “七八五” 工程银河亿 次机研制报告 [Report on the Development of YH-1 (785 project)]. Archives of College of Computer Science and Technology, NUDT, KTYH-I001/06.

10 “考虑到工程的周期与成本, 购买非核心部件是国际计算机工程领域的惯例, 美国 Cray-1 巨 型机大量部件甚至外围机等也都是购买的。”

11 “银河计算机是中国自行研制的第一台亿次电子计算机系统, 系统稳定可靠, 软件较为齐全, 其主要技术指标均达到和超过了鉴定大纲的要求, 具有国内先进水平, 某些方面达到了国际水平。 它的研制成功, 填补了国内巨型机的空白, 标志着中国进入了世界上研制巨型机的行列。……银河 计算机即将成为我国战略武器研制, 航天航空飞行器设计, 国民经济的预测和决策, 能源开发, 天 气预报, 图像处理, 情报分析, 以及各种科学研究的强大的计算工具。” 
The successful development of $\mathrm{YH}-1$ made China one of the few countries in the world that could develop supercomputers. In the book Tutorial: Supercomputer-Design and Application, published by the Institute of Electrical and Electronics Engineers, China's YH-1 is listed among the World Supercomputers (Hwang 1984).

\section{Research and innovation of YH-1: Comparison with Cray-1}

The supercomputer Cray-1 was developed by the US company Cray Research in March 1976. It is an array processor with a high processing speed and is characterized by pipeline and vector processing. The computing speed is more than 100 million calculations per second. A comparative analysis with Cray-1 shows the innovations of YH-1.

\subsection{Comparison of performance indexes}

Table 3: Comparison of main performance indexes between YH-1 and Cray-1

\begin{tabular}{|c|c|c|c|}
\hline & Items & YH-1 & Cray-1 \\
\hline \multirow{3}{*}{ Main parameters } & Dominant frequency & $20 \mathrm{Mc}$ & $80 \mathrm{Mc}$ \\
\hline & Operation speed (MFLOPS) & $40^{12}$ & 80 \\
\hline & $\begin{array}{l}\text { Number of instructions } \\
\text { (scalar/vector) }\end{array}$ & $140 / 57$ & $131 / 47$ \\
\hline \multirow{3}{*}{ Components } & Logical components & 2 ns: medium-scale & 0.5 ns: small-scale \\
\hline & Register & $\begin{array}{l}\text { Bipolar } 128 \times 1 \\
\quad 10 \mathrm{~ns}\end{array}$ & $\begin{array}{l}\text { Bipolar } 16 \times 1 \text {, } \\
6 \mathrm{~ns}\end{array}$ \\
\hline & Storage & $\begin{array}{l}\text { MOS } 16 \mathrm{~K} \times 1 \\
\quad 375 \mathrm{~ns}\end{array}$ & $\begin{array}{l}\text { Bipolar } 1 \mathrm{~K} \times 1 \\
\quad 50 \mathrm{~ns}\end{array}$ \\
\hline \multirow{4}{*}{ Pipelining } & Vector & $2 \times 3$ & 3 \\
\hline & Floating-point & $2 \times 3$ & 3 \\
\hline & Scalar & 4 & 4 \\
\hline & Address & 2 & 2 \\
\hline \multirow{3}{*}{ Registers } & Vector & $2 \times 8 \times 64 \times 64 b$ & $8 \times 64 \times 64 b$ \\
\hline & Scalar & $(8+64) \times 64 b$ & $(8+64) \times 64 b$ \\
\hline & Address & $(8+64) \times 24 b$ & $(8+64) \times 24 b$ \\
\hline \multirow{4}{*}{ Storages } & Maximum capacity & 4 million bytes & 1 million bytes \\
\hline & Actual capacity & 2 million bytes & 0.52 million bytes \\
\hline & Cross access module & 31 or 17 & 16 or 8 \\
\hline & Data traffic (scalar/vector) & $160 / 320 \mathrm{MB} / \mathrm{s}$ & $640 / 640 \mathrm{MB} / \mathrm{s}$ \\
\hline
\end{tabular}

“Yinhe jisuanji guojia jiandingshu” 银河计算机国家鉴定书 [National Appraisal of YH-1 Supercomputer]. Archives of College of Computer Science and Technology, NUDT, KTYH$1001 / 1$.

12 "FLOPS" refers to the number of floating-point results per second, while "calculations" refers to the number of machine instructions per second. Generally speaking, it takes about 3-5 instructions to get a floating-point result by scalar operation, so the operation speed of YH-1 is $40 \mathrm{M} \times 3$ (or 4) $\geq 100$ million calculations per second. 


\begin{tabular}{|c|c|c|c|}
\hline & & & Continued \\
\hline & Items & YH-1 & Cray-1 \\
\hline \multirow{9}{*}{$\begin{array}{l}\text { Associated } \\
\text { equipment }\end{array}$} & Instruction buffer station & $4 \times 64 \times 16 b$ & $4 \times 64 \times 16 b$ \\
\hline & Pairs of I/O channels & 12 & 12 \\
\hline & Total I/O transmission capacity & $320 \mathrm{MB} / \mathrm{s}$ & $640 \mathrm{MB} / \mathrm{s}$ \\
\hline & $\begin{array}{l}\text { Number of installed components } \\
\text { on plug-in }\end{array}$ & 110 (at most) & 288 (at most) \\
\hline & Number of plug-in types & 606 & 1662 \\
\hline & Cabinet type & Cylinder & Cylinder \\
\hline & The number of wedge machines & 7 & 12 \\
\hline & Cooling mode & Air-cooled & Freon liquid cooling \\
\hline & Power consumption & $25 \mathrm{~kW}$ & $115 \mathrm{~kW}$ \\
\hline \multirow{7}{*}{ Soft } & Operating systems & YHOS & $\cos$ \\
\hline & Assemblers & YHAL & CAL \\
\hline & Compiler system & YHFT & CFT \\
\hline & \multirow{2}{*}{ Mathematical subroutine library } & 80 categories; & 41 categories; \\
\hline & & 292 modules & 82 modules \\
\hline & Diagnostic procedures & $\begin{array}{l}\text { Component level and } \\
\text { plug-in level diagnosis }\end{array}$ & Diagnostic procedures \\
\hline & Vector recognizer & $\sqrt{ }$ & $x$ \\
\hline \multicolumn{2}{|c|}{ Mean time between failures (MTBF) } & 441 hours & 159.2 hours \\
\hline
\end{tabular}

\subsection{Comparison of advantages and disadvantages}

\subsubsection{The advantages of Cray-1 over YH-1}

The architecture of Cray-1 is the model for many later supercomputers in the world, including $\mathrm{YH}-1$. The development of $\mathrm{YH}-1$ mainly refers to the design idea of Cray-1, but the technical details of Cray-1 implementation are not known. Cray-1 was successfully developed in 1975, and YH-1 did not come out until 1983. Under ideal conditions, the operation speed of Cray-1 can reach four times that of $\mathrm{YH}-1$. Therefore, Cray-1 is better than $\mathrm{YH}-1$ in architecture.

In terms of hardware, the Cray-1 logic module uses the high-precision small-scale integrated circuit first invented by Fairchild Semiconductor in the world at that time, which made the working frequency of Cray-1 an astonishing $80 \mathrm{Mc}$ (the highest in the world at that time). However, due to the international blockade and its own technological level, the main frequency of YH-1 can only reach $20 \mathrm{Mc}$, far less than that of Cray-1. Cray-1 I/O has a total transmission capacity of $640 \mathrm{MB} / \mathrm{s}$, and YH-1 is only half of that $-320 \mathrm{MB} / \mathrm{S}$. Therefore, Cray-1 is better than $\mathrm{YH}-1$ in hardware.

In terms of software, Cray- 1 is a self-developed operating system, compiler, and assembler. YH-1 also uses its own operating system, compiler, and assembler, but it is required to be compatible with the Cray-1 software system, which is a small advantage 
of Cray-1 over $\mathrm{YH}-1$ in software.

In terms of engineering, the mechanical structure of the wedge-shaped frame of the cylinder cabinet, and the high-density assembly process of the large plug-in board structure of Cray-1 were the first in the world. YH-1 is based on Cray-1, but the number of frames, the number of plug-ins on the rack, the types and quantity of components on the plug-in, and the engineering level of the whole machine are not as good as Cray-1. Cray-1 is a Freon liquid cooling system, which cannot be realized by the technology or conditions of YH-1. It can only use the air-cooling system with static pressure and a short air path. Thus, Cray-1 is superior to $\mathrm{YH}-1$ in cooling mode.

\subsubsection{The advantages of YH-1 over Cray-1}

\subsubsection{Innovation of architecture}

The CPUs of Cray-1 and YH-1 are both single processors, but Cray-1 is a vector single unit processing structure. $\mathrm{YH}-1$ first proposed a bi-vector array component structure scheme, that is, six pipelines of double floating point operation unit, six pipelines of bivector component, and bi-vector quantity register, which can obtain two operation results per beat.

YH-1 has designed full pipelined functional components and composite pipeline technology. Cray-1 has twelve functional components, while $\mathrm{YH}-1$ has eighteen, all of which are pipeline structure. Instruction control and data access also adopt pipelining mode. In addition, composite pipeline technology is employed to solve the problems related to vector instructions and to improve the parallelism of components, so as to enhance the actual operation speed.

Compared with Cray-1, YH-1 adds "compression-restore" transfer instruction and indirect address transfer instruction, which provides convenience for some algorithms, greatly saving space and significantly accelerating the operation speed.

This architectural innovation makes it possible for $\mathrm{YH}-1$ to achieve more than 100 million times operation even if it cannot reach the ultra-high clock frequency of Cray-1.

\subsubsection{Improvement of hardware design}

Since 1978, the module with a storage of $400 \mathrm{~ns}$ and $64 \mathrm{~KB}$ has been successfully developed for $\mathrm{YH}-1$ through using the MOS with a storage cycle of 375 ns. The structure of the storage system is multi-module, prime-analog, dual-bus, and crossaccess. This structure can reduce the probability of access conflict and meet the demand of data flow in bi-vector array operation. ${ }^{13}$ The storage control technology of dual bus

13 “Yinhe-I youguan yingjian sheji ziliao" 银河 - I 有关硬件设计资料 [Information on Hardware Design of YH-1]. Archives of College of Computer Science and Technology, NUDT, KTYH-I017. 
cross access of prime modulus (31/17) includes fast address transformation algorithm and its implementation, access conflict handling, and queue structure, which ensures the realization of high-speed data flow. However, Cray-1 did not use the 16-K MOS module for logic components until it launched Cray-1M in 1982. The actual capacity of Cray-1's main memory is 0.52 million bytes and the maximum capacity is one million, which are not as good as YH-1's two million and four million bytes, respectively; Cray1 's 16/8 cross access module structure could easily cause conflicts in high-speed

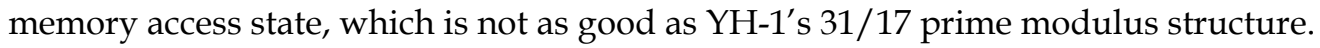

YH-1 improves the Cray-1 floating-point reciprocal approximate iterative algorithm and simplifies the structure of components. The equipment of $\mathrm{YH}-1$ is saved by $60 \%$ compared with Cray-1 with the same accuracy, and the pipeline is reduced from fourteen stations to six, which shortens the starting time of calculation. YH-1 has a micro-command library in the instruction flow control unit, which simplifies the control and saves the equipment. YH-1 also adopts the dual buffer disk control interface operated by micro-programing to give full play to the disk transmission speed; the corresponding hardware interface is designed according to the characteristics of the peripheral machine.

The new technology, which partly improves and breaks through Cray-1 design, is adopted to ensure the high speed and high performance of $\mathrm{YH}-1$.

\subsubsection{Breakthrough of software system}

YHOS, the operating system of $\mathrm{YH}-1$, is a distributed batch processing system independently researched and developed. The mainframe can undertake 128 jobs, and 63 of them can be put into operation at the same time. It allows users to specify a prioritized job, which can be given preference of various system resources. It also allows users to save records in real time before going off the system or conduct debugging before starting the system to implement the users' subsection calculation. YHOS adopts the program design of hierarchical modular structure. The functions of each module are relatively independent, the interface is clear, and the expansibility is strong. The function of YHOS is more complete than that of COS, the operating system of Cray-1.

YHFT, YH-1 vector high-level language, uses three-level optimization techniques, such as basic block optimization, expression optimization, and object code optimization, to make full use of the hardware efficiency of YH-1. YHFT is also equipped with a vector recognizer, and the vector analysis and recognition method, which combine the new subscript tracking method and the traditional coordinate method, is adopted to enhance the recognition function. After completion, it can recognize the DO loop body that contains GOTO statement and three kinds of IF statements, so that it can also be vectorized. In contrast, Cray-1 has neither vector recognizer nor its corresponding 
function.

The mathematical subroutine library of YH-1 contains not only all FORTRAN internal functions, but also various vector subroutines, up to 80 categories and 292 modules. Due to the in-depth research on parallel algorithm and parallel program design, it not only has more functions and quantity, but also has higher precision and speed than Cray-1. ${ }^{14}$

In summary, though YH-1 is reliant on Cray-1's basic software, YH-1's language, operating system, and optimization techniques are more user-friendly and allow for more choice.

\subsubsection{Advantages of engineering technology}

\section{(1) Maintenance diagnosis technology}

YH-1 conducts dual array components comparison, double data path comparison, double sets of key control components comparison, and parity check to carry out a comprehensive testability layout. Compared with the overall system, the hardware only increases by $5 \%$ in quantities, designs, and processes. Most faults (including instantaneous faults) can be detected in time, which creates a very favorable condition for plug-in level fault diagnosis.

The main memory of YH-1 adopts the Hamming code check system, which can correct one dislocation and detect multiple errors and is better than Cray-1's parity check. It greatly boosts its reliability, and it has complete self-test means that can significantly facilitate maintenance. In particular, large amounts of important information (including access source, body number, internal address, and error correction code) of the main memory verification system can be recovered by software, so as to ensure the accurate location of memory failure. After using the Hamming check system, the mean time between failures (MTBF) of YH-1's main memory is increased by about 4.6 times, and the reliability is increased by about 9 times, which is superior to Cray-1.

YH-1 has independently established a three-level diagnosis system at the component, plug-in, and module levels, with fast diagnosis speed, accurate positioning, and convenient use. The host plug-in level diagnosis can isolate most of the fixed logic faults to three plug-ins, which greatly shortens the maintenance time of YH-1. Through the unit centralizing diagnosis and processing, under the condition that the hardware structure remains basically unchanged, all kinds of control information (including clock control) can be swept in and out from the diagnosis and maintenance machine to the host computer, and all the hard check information can be recovered, which provides a means for the diagnosis and automatic adjustment of other machines. At the same time, 
a variety of fault diagnosis generation software is developed, such as DDL-D and its translator, random code generation technology, test code generation algorithm, logic fault simulation algorithm, and weight " 3 " compression map fault dictionary, which effectively solves the test generation problem of complex functional components and logic plug-ins. By contrast, Cray-1 has no hardware monitoring system, which can easily cause hardware failure and problems. ${ }^{15}$

\section{(2) Manufacture technology of mainframe computer}

The YH-1 researchers worked closely as a team to tackle key problems. Hundreds of thousands of machine components underwent strict aging tests and screening. There are about 5000 metallized holes on each of hundreds of multilayer printed boards and base plates. All of the holes passed resistance tests on the hole-wall. There are no missing solder joints, false soldering, and hanging tin in the over 2.3 million solder joints.

\section{(3) Low-voltage high-current unstable DC power supply technology}

The technicians of $\mathrm{YH}-1$ have solved key problems, involving parallel feeding of six phase and twelve phase high-power low-voltage transformer, for example. This marks the first use of the parallel power feed system of an AC voltage stabilizing and rectifying filter on the computer in China. It has high efficiency and high reliability, the voltage difference of each point of the whole machine is small, and the maximum power consumption is $25 \mathrm{~kW}$, which improves the stability and reliability of the system. In comparison, Cray-1 is a simple and extensive power supply with high current and high power consumption. The maximum power consumption of Cray-1 is $115 \mathrm{~kW}$, which is almost five times that of $\mathrm{YH}-1$, so its energy consumption rate is very high.

When Cray-1 first began operating at the Los Alamos Scientific Laboratory of the United States (in May and June of 1976), engineering and technological shortcomings were still being resolved. For example, the MTBF was only 2.5-7.5 hours. Until 1981, the MTBF of Cray-1 was stabilized to 159.2 hours according to the statistical results; in comparison, the MTBF of $\mathrm{YH}-1$ during its initial development was already 441 hours.

\section{Inspirations from the development of YH-1}

YH-1 is one of the successful cases of China's Big Science Projects, and the experience and lessons of its development are of certain practical significance.

15 The Cray-1 developer, Seymour Cray, was so confident that he did not set up a hardware monitoring system for Cray-1. For the errors that Cray-1 encountered in its first six months, Clay always thought that it was vulnerable to cosmic rays. Later, he was initially reluctant to include error-detecting mechanisms, but eventually did so, adding what became known as parity memory (Brazil 2021). 


\subsection{Strategic support from national leaders}

YH-1 was successfully developed with the attention and support of China's top leadership, which reflects the demands of the nation, strategic will, and strategic value of developing supercomputers. Deng Xiaoping personally assigned the task of developing supercomputers. As the highest leading institution in science and technology of defense, COSTND was responsible for supervision.

NUDT took on the development of YH-1 as its primary project and was supported by a network of universities, institutes, offices, and professional groups, each with their own responsibilities. The project demanded close cooperation and participating institutions held regular joint meetings to address various problems in a timely manner. At the same time, YH-1 was strongly supported by the state in terms of human, financial, and material resources. In the late 1970s, the "Cultural Revolution" had just ended, and the whole nation had to start from scratch. Although the 1976 Tangshan Earthquake brought great damage to the national economy, the state still allocated huge funds to ensure the development of $\mathrm{YH}-1$. The relevant state departments also assisted in the research and development units and enriched the YH-1 research team by recruiting technical personnel from scientific research institutes nationwide, employing university graduates majoring in computer science, and recruiting outstanding soldiers and educated youth. The state's all-round support enabled the YH-1 project to be completed with high speed, high quality, and high efficiency.

\subsection{Scientific research and management mechanism of concentrating on major tasks}

“Concentrating our forces on major tasks" (集中力量办大事) is the basic principle guiding large-scale scientific projects under the socialist system in China. It had been successfully implemented in the “Two Bombs and One Satellite" (两弹一星) project, and it also guided the development of the $\mathrm{YH}-1$ supercomputer.

YH-1 was a complex large-scale system, which required the team to cooperate closely to form a group operation. The chief designer carried out the top-level design and overall planning, and divided it into several subsystem tasks, which were then assigned to director designers. Each director designer divided the sub-system task into several sub tasks, which were undertaken by heads of each research group. This kind of engineering research system required a high degree of internally centralized cooperation to form a link as a whole to effectively promote scientific research. The centralized organization of this project meant that the leaders of smaller groups could easily discuss problems collectively and reach coordinated solutions in a timely manner. The organization also allowed for a clear representative of each department. If a 
technical problem arose, the representative could be quickly summoned for troubleshooting.

The development of $\mathrm{YH}-1$ was only challenging because it was large and involved a wide range of fields. In this case, the national cooperation in scientific research played an important role. Thirty-seven brother units in China, such as CAS, Tsinghua University, Wuhan University, Fudan University, and Hunan University, provided strong technical support.

\subsection{Application mode tightly tied with users}

Before the development of YH-1, Chief Designer Ci Yungui conducted in-depth research twice across the whole country, and concluded that there was an urgent need in national defense construction and in the economy for a supercomputer. He reported his findings to the central government and strived to carry out the task as soon as possible. After the $\mathrm{YH}-1$ project began, the research team focused on how to break through the key technology and successfully developed the first supercomputer in China thanks to strong support from the government and army. However, the practical application of YH-1 was ignored. For example, the YH-1 drawing software was not developed synchronously, so it could not meet the urgent needs of some user processing large amounts of data; the lack of scientific databases and special packages that combined with users' commands led to the difficulty in vector computing efficiency of some users' own applications on YH-1; the research on parallel algorithm and parallel programing needed to be carried out vigorously; the research and construction of the local network system of $\mathrm{YH}-1$ did not keep up in time; and the application scope and user units were not further expanded.

In order to make up for the shortcomings in application following project completion, NUDT signed an agreement with the Bureau of Geophysical Prospecting (BGP) of the Ministry of Petroleum Industry (MPI) and the Chinese Academy of Engineering Physics to carry out the application cooperation in data processing of petroleum seismic exploration and nuclear weapons research. ${ }^{16}$ Three YH-1 supercomputers were produced: one at NUDT, one as the host computer of "the $\mathrm{YH}$ seismic data processing system" at the BGP research institute of the MPI in Hebei Province, and one as the host computer of the Southwest Computing Center in Sichuan Province. All of these made contributions to national defense construction and economic development, but YH-1's lagged application services affected the development of $\mathrm{YH}-2$. After YH-1 was successfully developed, it took another five years for the YH-2 project to be approved because of the lack of clear user requirements. In order to learn from the

16 “Yinhe-I jinqi youguan hetong” 银河 - I 近期有关合同 [Recent Contracts of YH-1]. Archives of College of Computer Science and Technology, NUDT, KTYH-1034/6. 
lessons, before the development of YH-3, NUDT carried out feasibility research of application, seeking a demand that the state pays close attention to and continues to exist. Finally, it selected the nuclear application field, and established a close "binding" cooperative relationship with the important users of nuclear weapons development and nuclear science research of the national army. The future development and application of YH series supercomputers have found the right way.

\section{Conclusion}

The development and innovation of China's first supercomputer, YH-1, reflects the scientific research process of a significant number of national large-scale projects in science and engineering at the beginning of the reform and opening-up period. Its development stemmed directly from the strategic needs of the country and the army, created key technologies, and adhered to the road of independent innovation with Chinese characteristics. The success of $\mathrm{YH}-1$ benefits not only from the historical opportunities and development conditions of the reform and opening-up, but also from the continuous exploration and accumulation of research institutions and workers in relevant science and technology since the founding of the PRC, from the assistance and technology transfer of the Soviet Union in the early stages of the computer industry, from the successful mechanism operation and spiritual incentive of "Two Bombs and One Satellite," and from other large scientific projects. YH-1 met the urgent need of China's cutting-edge technology research, supported the national economic construction, broke through the technological blockade of the West, made China stride forward in mastering computer science and technology, and accumulated experience in developing high-performance computers for the country. Forty years after the reform and opening-up, China's supercomputers have become world-class examples. The history of their development provides beneficial enlightenment, especially under the fierce modern Sino-US competition in high-end science and technology.

\section{References}

Bai, Ruixue 白瑞雪, Wu Jianjun 吴建军, and Si Hongwei 司宏伟. 2013. “Rang 'chao suan meng' zhutui “Zhongguo meng': Jujiao Tianhe Erhao jisuanji” 让 “超算梦” 助推 “中国梦” ——聚焦 天河二号计算机 [Let "Supercomputing Dream" Boost “China Dream": Focus on TH-2 Computer]. Xinhua News Agency, June 17, 2013.

Brazil, Rachel. 2021. “Supercomputers Face a Cosmic Challenge." Physics World 34 (7): 30.

Ci, Yungui 慈云桂, and Hu Shouren. 1980. “785 xing jisuanji zongti sheji” 785 型计算机总体设计 [General Design of Computer 785]. Jisuanji gongcheng yu kexue 计算机工程与科学 [Computer Engineering and Science], no. 4, 3-4.

$\mathrm{Hu}$, Shouren 胡守仁. 2016. Jisuanji jishu fazhanshi (yi) 计算机技术发展史（一） [History of 
Computer Technology: Vol. 1]. Changsha: National University of Defense Technology Press. Hwang, K. 1984. Tutorial: Supercomputers - Design and Applications. N.p.

Liu, Shi'en 刘世恩. 1996. Yinhe song 银河颂 [Ode to the YH-2]. Changsha: Hunan Publishing House.

Liu, Shi'en. 1998. Yinhe song (xu) 银河颂（续） [Ode to the YH-3]. Changsha: Hunan Science and Technology Press.

Liu, Wentao 刘文蹈, and Si Hongwei. 2009. “Woguo shoutai qianwanyici chaoji jisuanji yanzhi chenggong” 我国首台千万亿次超级计算机研制成功 [China's First Quadrillion-Times Supercomputer]. Hunan ribao 湖南日报 [Hunan Daily], October 29, 2009, 1.

Liu, Yidong 刘益东, and Li Genqun 李根群. 2006. Zhongguo jisuanji chanye fazhan zhi yanjiu 中国 计算机产业发展之研究 [Research on the Development of China's Computer Industry]. Jinan: Shandong Education Press.

Murray, Charles J. 1997. The Supermen: The Story of Seymour Cray and the Technical Wizards Behind the Supercomputer. New York: John Wiley \& Sons.

School History Editorial Board of NUDT 国防科大校史编审委员会. 1993. Guofang Keji Daxue xiaoshi (1953-1993) 国防科技大学校史（1953 1993） [The History of National University of Defense Technology (1953-1993)]. Changsha: National University of Defense Technology Press. School History Editorial Board of NUDT. 1994. Guofang Keji Daxue Jisuanjixi jian Yanjiusuo shi (1956-1993) 国防科技大学计算机系兼研究所史（1956 1993) [The History of the Department and Institute of Computer Science, National University of Defense Technology (1956-1993)]. Internal printing.

Si, Hongwei, and Feng Lisheng 冯立昇. 2017. “Zhongguo diyitai yici juxing jisuanji 'yinhe-I' yanzhi licheng ji qishi” 中国第一台亿次巨型计算机 “银河-I” 研制历程及启示 [The Development of the First Supercomputer "YH-1" in China and Its Inspiration]. Ziran kexueshi yanjiu 自然科学史研究 [Studies in the History of Natural Sciences] 36 (4): 563-580.

Xu, Zuzhe 徐祖哲. 2015. Suyuan Zhongguo jisuanji 溯源中国计算机 [Tracing the Origins of Computers in China]. Beijing: SDX Joint Publishing.

Zhang, Jiuchun, and Zhang Baichun. 2007. "Founding of the Chinese Academy of Sciences' Institute of Computing Technology." IEEE Annals of the History of Computing, no. 3, 16-33.

Zhao, Yanghui 赵阳辉. 2012. “Yinhe yici juxing jisuanji chuangxinxing yanjiu: Yi gongcheng benzhi juece ji wenhua wei zhongxin" 银河亿次巨型计算机创新性研究一一工程本质、决策 及文化为中心 [Study of the Innovation of YH-1 Supercomputer: Focusing on Engineering Nature, Decision-Making and Culture]. Ziran bianzhengfa yanjiu 自然辩证法研究 [Studies in Dialectics of Nature] 28 (7): 51-55.

Zhou, Xingming 周兴铭, and Zhao Yanghui. 2005. “Ci Yungui yu Zhongguo yinhe ji yanjiu qunti de fazhan licheng” 慈云桂与中国银河机研究群体的发展历程 [Ci Yungui and the Development of Chinese "YH Research Group"]. Zhongguo kejishi zazhi 中国科技史杂志 [The Chinese Journal for the History of Science and Technology] 26 (1): 37-45. 